\title{
Article \\ Estimating the Polytropic Indices of Plasmas with Partial Temperature Tensor Measurements: Application to Solar Wind Protons at $\sim 1$ au
}

\author{
Georgios Nicolaou ${ }^{1, *(D)}$, George Livadiotis ${ }^{1}$ and Mihir I. Desai ${ }^{1,2}$ \\ 1 Southwest Research Institute, San Antonio, TX 78238, USA; george.livadiotis@swri.org (G.L.); \\ mihir.desai@swri.org (M.I.D.) \\ 2 Department of Physics and Astronomy, University of Texas at San Antonio, San Antonio, TX 78249, USA \\ * Correspondence: georgios.nicolaou@swri.org
}

Citation: Nicolaou, G.; Livadiotis, G. Desai, M.I. Estimating the Polytropic Indices of Plasmas with Partial Temperature Tensor Measurements: Application to Solar Wind Protons at $\sim 1$ au. Appl. Sci. 2021, 11, 4019. https://doi.org/10.3390/app11094019

Academic Editor: Emilio Martines

Received: 20 March 2021

Accepted: 22 April 2021

Published: 28 April 2021

Publisher's Note: MDPI stays neutral with regard to jurisdictional claims in published maps and institutional affiliations.

Copyright: (C) 2021 by the authors. Licensee MDPI, Basel, Switzerland. This article is an open access article distributed under the terms and conditions of the Creative Commons Attribution (CC BY) license (https:// creativecommons.org/licenses/by/ $4.0 /)$.

\begin{abstract}
We examine the relationships between temperature tensor elements and their connection to the polytropic equation, which describes the relationship between the plasma scalar temperature and density. We investigate the possibility to determine the plasma polytropic index by fitting the fluctuations of temperature either perpendicular or parallel to the magnetic field. Such an application is particularly useful when the full temperature tensor is not available from the observations. We use solar wind proton observations at $\sim 1$ au to calculate the correlations between the temperature tensor elements and the scalar temperature. Our analysis also derives the polytropic equation in selected streamlines of solar wind plasma proton observations that exhibit temperature anisotropies related to stream-interaction regions. We compare the polytropic indices derived by fitting fluctuations of the scalar, perpendicular, and parallel temperatures, respectively. We show that the use of the parallel or perpendicular temperature, instead of the scalar temperature, still accurately derives the true, average polytropic index value, but only for a certain level of temperature anisotropy variability within the analyzed streamlines. The use of the perpendicular temperature leads to more accurate calculations, because its correlation with the scalar temperature is less affected by the anisotropy fluctuations.
\end{abstract}

Keywords: space plasmas; solar wind; thermodynamics

\section{Introduction}

\subsection{Temperature Anisotropy}

The analysis of plasma particle velocity distribution functions (VDFs) is necessary to understand the kinetic and thermodynamic properties of plasmas. The statistical moments of the VDFs determine the plasma bulk properties, such as the plasma density, velocity and temperature. The plasma temperature, specifically, is analogous to the average kinetic energy of the plasma particles in the reference frame of the plasma and is calculated by the second order velocity moment of the plasma VDF. In several cases, the VDFs of space plasmas exhibit temperature anisotropies, which are organized by the magnetic field direction (e.g., [1-3]). In these cases, the complete description of plasmas requires the knowledge of the temperature perpendicular to the magnetic field $T_{\perp}$ and the temperature parallel to the magnetic field $T_{\|}$. In order to resolve the full temperature tensor from the data analysis, we need observations of full, three-dimensional (3D) VDFs or observations of 2D pitch-angle distributions of the plasma particles [4,5].

Several studies discuss heating/cooling mechanisms that result in the anisotropic heating of plasmas. For instance, [6] show analytically how the temperature anisotropy is modified across shock waves, accounting for observations of interplanetary and planetary bow shocks. The analysis by [7] shows a perpendicular heating of solar wind ions as they cross the bow Shock of Venus. The anisotropic heating is stronger closer to Venus, where 
the shocked solar wind interacts with the planet's ionosphere. In another example, [8] examine ARTEMIS ion observations of a long-duration magnetotail exhaust. The authors show that the parallel temperature dominates far away from the $x$-line, indicating strong parallel ion heating. Near the neutral plane, however, the perpendicular temperature dominates, indicating that ions undergo a Speiser-like motion.

In gyro-tropic plasmas, the scalar temperature $T$ is the trace of the temperature tensor:

$$
T=\frac{1}{3} T_{\|}+\frac{2}{3} T_{\perp}
$$

and the temperature anisotropy is the ratio:

$$
\alpha=\frac{T_{\perp}}{T_{\|}} .
$$

Unfortunately, in situ observations are not always optimal to construct 3D VDFs or full pitch-angle distributions from which we can determine the full temperature tensor. For instance, in some occasions, Faraday cup observations obtain 1D VDFs from which we can derive the temperature along one direction only [9]. Therefore, here, we would like to explore the correlations between the temperature tensor elements, the scalar temperature, and the temperature anisotropy. These correlations are useful to understand what plasma dynamics information we lose when accurate temperature measurements occur in only one direction. By combining Equations (1) and (2), we express the scalar temperature as a function of $\alpha$ and $T_{\perp}$ :

$$
T=\frac{1}{3 \alpha} T_{\perp}+\frac{2}{3} T_{\perp}=T_{\perp}\left[\frac{1}{3}\left(\frac{1}{\alpha}+2\right)\right] \Rightarrow \log T=\log T_{\perp}+\log \left(\frac{1+2 \alpha}{3 \alpha}\right),
$$

and as a function of $\alpha$ and $T_{\|}$:

$$
T=\frac{1}{3} T_{\|}+\frac{2}{3} \alpha T_{\|}=T_{\|}\left(\frac{1+2 \alpha}{3}\right) \Rightarrow \log T=\log T_{\|}+\log \left(\frac{1+2 \alpha}{3}\right) .
$$

We would like to examine how the above relationships apply in solar wind proton observations, and how well $T_{\perp}$ or $T_{\|}$can be used to study a specific thermodynamic process; the polytropic process.

\subsection{Polytropic Process}

The polytropic process describes the transition of a fluid from one thermodynamic state to another through the relationship between the fluid density and temperature (or pressure $[10,11])$. Through the polytropic equation, we achieve a closure between the higher order moments and the plasma density. In terms of plasma density $n$ and temperature $T$, the polytropic equation is:

$$
T=C n^{\gamma-1},
$$

where $C$ is a constant characterizing specific streamlines, and $\gamma$ is the polytropic index. The value of $\gamma$ is indicative for the energy transfer during the process. The determination of $\gamma$ in several applications is useful in understanding the physical mechanisms in the system. We take the logarithm of Equation (5):

$$
\log (T)=(\gamma-1) \log (n)+\log C,
$$

so that $\gamma$ is determined by the slope of the linear relationship between $\log (T)$ and $\log (n)$. The polytropic index is a useful tool to study dynamic plasma fluctuations [12] and describes the heating/cooling of the plasma without the necessity to solve the energy equation, which can be very complicated [13]. Several studies analyze plasma observations and determine the polytropic index of different species in several regions through the heliosphere. 
For instance, [14] determine the polytropic index of plasma electrons in the Saturnian magnetosphere from Cassini observations, while [15] derive the polytropic index of energetic plasma ions in the magnetosphere of Saturn, observed by the same spacecraft. The studies by $[16,17]$ use a polytropic model to characterize the temperature and density fluctuations in the distant Jovian magnetosheath and boundary layer. In other examples, [18,19] determine the polytropic expansion of the solar wind protons in the inner heliosphere, while [20-22] calculate $\gamma$ of protons at $\sim 1$ au. Elliott et al. [23] calculate $\gamma$ of solar wind protons through the outer heliosphere, and [24] determines the polytropic process in the inner heliosheath. Finally, [25] discuss the radial profile of the polytropic index within the heliosphere.

As mentioned in the previous subsection, in situ plasma measurements cannot always determine the full temperature tensor. Therefore, we would like to determine the polytropic relationship as a function of the temperature in a single direction. For simplicity, we investigate the polytropic relationship with $T_{\perp}$ and $T_{\|}$, although we could generalize for any direction. By combining Equations (3), (4) and (6), we can express the polytropic equation in terms of $T_{\perp}$ and $\alpha$ :

$$
T_{\perp}=C\left(\frac{3 \alpha}{2 \alpha+1}\right) n^{\gamma-1} \Rightarrow \log \left(T_{\perp}\right)=\log C+\log \left(\frac{3 \alpha}{2 \alpha+1}\right)+(\gamma-1) \log (n),
$$

or in terms of $T_{\|}$and $\alpha$ :

$$
T_{\|}=C\left(\frac{3}{2 \alpha+1}\right) n^{\gamma-1} \Rightarrow \log \left(T_{\|}\right)=\log C+\log \left(\frac{3}{2 \alpha+1}\right)+(\gamma-1) \log (n) .
$$

According to Equations (7) and (8), we can determine a linear relationship between either $\log \left(T_{\perp}\right)$ and $\log (n)$, or $\log \left(T_{\|}\right)$and $\log (n)$, only if $\alpha$ does not vary within the examined interval. In both cases, the slope of the linear model defines $\gamma$. In other words, if we use a temperature element along one direction only and we do not know the temperature anisotropy, we can derive the polytropic index only in intervals with nearly constant temperature anisotropy.

This paper examines the polytropic behavior of solar wind protons in an annual interval, while a future study will expand the analysis in a dataset covering more than 25 years of observations. Our selected time period shows clear evidence of anisotropic heating within stream-interaction regions. We determine the polytropic index by applying three different fittings: one using the scalar temperature, one using the perpendicular temperature $T_{\perp}$, and one using the parallel temperature $T_{\|}$. We compare the derived polytropic indices and discuss their similarities and differences. This paper is organized as follows. In the next section, we explain the data we use. Section 3 describes our analysis methods. In Section 4, we show our results, which are discussed in Section 5. Finally, Section 6 summarizes our conclusions.

\section{Data}

We use 92 s resolution proton plasma observations obtained by the Wind spacecraft [26] at a heliocentric distance $~ 1$ au in 1995 (see Figure 1). We use the proton plasma density $n$ and its 1-sigma uncertainty $\sigma_{n}$, the bulk speed $V$, the scalar thermal speed, and the thermal speed perpendicular and parallel to the magnetic field and their uncertainties. From these, we calculate the scalar temperature $T$, the perpendicular temperature $T_{\perp}$, the parallel temperature $T_{\|}$, and their 1-sigma uncertainties $\sigma_{T}, \sigma_{T, \perp}$, and $\sigma_{T, \|}$, respectively. We use the plasma bulk parameters, which are derived from Maxwellian fits to the observed velocity distribution functions [27]. The goodness of each fit is quantified by a chi-squared $\left(\chi^{2}\right)$ value. In order to increase the statistical significance of our analysis, we exclude data points with $\chi^{2}>5$. Additionally, we only analyze data points with relative uncertainties $\frac{\sigma_{n}}{n}$, $\frac{\sigma_{T}}{T}, \frac{\sigma_{T, \perp}}{T_{\perp}}$ and $\frac{\sigma_{T, \|}}{T_{\|}}$, all smaller than $30 \%$. Finally, for the calculation of the Bernoulli integral 
(see Section 3), we use magnetic field $B$ measurements, averaged over the time resolution of the plasma data.
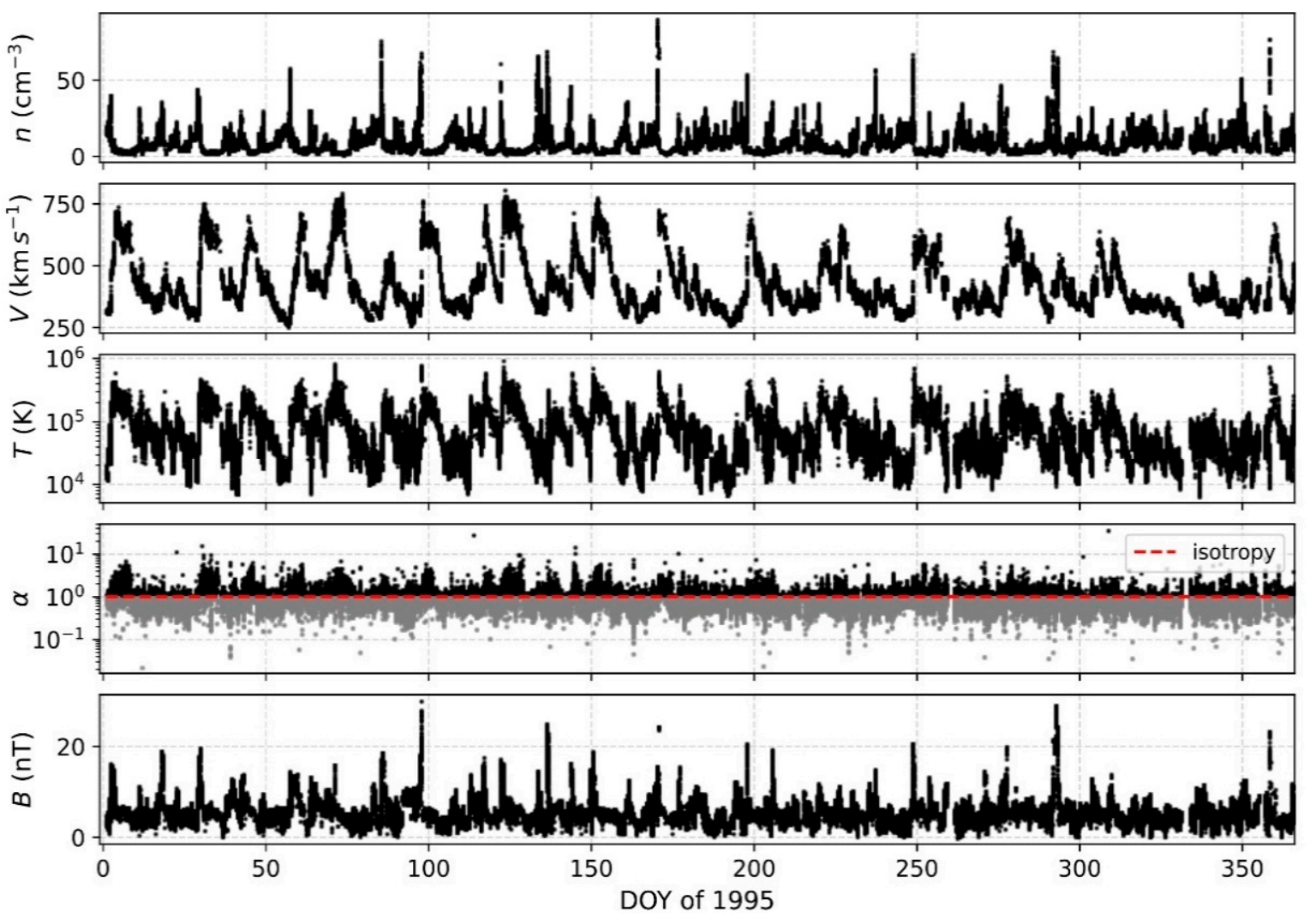

Figure 1. Time series of solar wind proton bulk parameters as measured by the Wind spacecraft in 1995. From top to bottom, we show the proton number density, speed, scalar temperature, temperature anisotropy, and the magnetic field.

\section{Methodology}

We initially investigate the temperature anisotropy as a function of the bulk speed by examining the histograms of the two parameters. We also calculate the linear Pearson correlation coefficients between $T$ and $T_{\perp}, T$ and $T_{\|}$, and $T_{\perp}$ and $T_{\|}$. We calculate for slow $\left(V<500 \mathrm{kms}^{-1}\right)$ and fast $\left(V>500 \mathrm{kms}^{-1}\right)$ solar wind. Additionally, in order to investigate the large-scale correlations between the plasma density and temperature, we plot the $2 \mathrm{D}$ histograms of $\log (n)$ and $\log (T), \log (n)$ and $\log \left(T_{\perp}\right)$, and $\log (n)$ and $\log \left(T_{\|}\right)$.

We then group the entire time series in subintervals of eight consecutive measurements, which cover $\sim 12 \mathrm{~min}$ if there are no data gaps between the data points. We calculate the standard polytropic index $\gamma$ and its uncertainty $\sigma_{\gamma}$ in each subinterval by fitting the polytropic model to $\ln (T)$ vs. $\ln (n)$ observations. For the fitting, we use an orthogonal distance regression. The weighted fitting uses $\frac{\sigma_{T}}{T}$ and $\frac{\sigma_{n}}{n}$ as the 1-sigma uncertainties of the fitted $\ln (T)$ and $\ln (n)$ data points, respectively. We further quantify the homogeneity of the plasma within each subinterval by calculating the ratio of the standard deviation of the Bernoulli integral over its absolute mean value [20-22,28,29]. We exclude time sub-intervals in which this ratio is larger than 0.1. Finally, we repeat the linear regression method to determine the polytropic indices from the slopes between $\ln (n)$ and $\ln \left(T_{\perp}\right)$, and between $\ln (n)$ and $\ln \left(T_{\|}\right)$.

\section{Results}

According to Figure 1, Wind observed several stream-interaction regions in 1995. Within these regions, the observed bulk velocity increases to $V \sim 750 \mathrm{kms}^{-1}$ over the 
background solar wind speed $V \sim 300 \mathrm{kms}^{-1}$. The scalar temperature also increases by an order of magnitude within the interaction regions. The temperature anisotropy seems to increase with increasing speed and temperature. In the bottom left panel of Figure 2, we show the 2D histogram of $\log (\alpha)$ and $V$, as observed in 1995. We normalize the occurrence in each column ( $V$ bin) to its maximum value. The top panel of Figure 2 shows the 1D histogram of $V$, while the right panel shows the 1D histogram of $\log (\alpha)$ for the same interval. Although the most frequent temperature anisotropy is below 1, there is a sharp increase at $V \sim 600 \mathrm{kms}^{-1}$. The peak of the most frequent value (magenta) is $\alpha \sim 2.5$. The anisotropy decreases again for $V>650 \mathrm{kms}^{-1}$. We interpret this as an anisotropic heating/cooling of the plasma protons within stream-interaction regions.

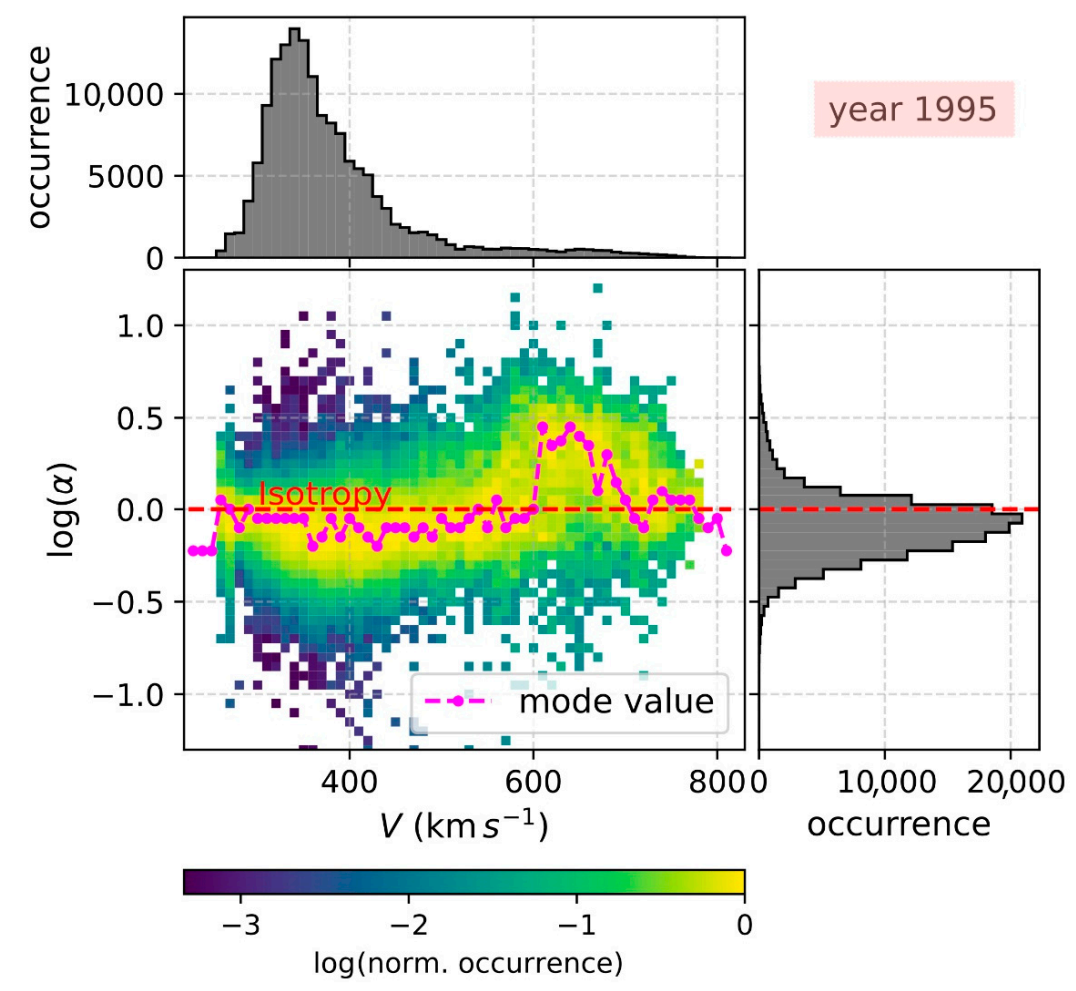

Figure 2. Two-dimensional histogram of $\log (\alpha)$ and $V$, normalized to the maximum value in each column ( $V$ bin). The magenta line shows the most frequent (mode) value in each column. In the top panel, we show the 1D histogram of $V$; in the right panel, we show the 1D histogram of $\log (\alpha)$.

The top panels of Figure 3 show scatter plots of the logarithms of the temperature tensor elements and the scalar temperature in the slow solar wind $\left(V<500 \mathrm{kms}^{-1}\right)$, while the bottom panels show the corresponding plots for the fast solar wind $\left(V>500 \mathrm{kms}^{-1}\right)$. In each panel, we plot the identity line $(y=x)$ and show the calculated linear Pearson correlation coefficient of the parameters. We observe that in the slow solar wind, $\log (T)$ is strongly correlated with both $\log \left(T_{\perp}\right)$ and $\log \left(T_{\|}\right)$. The Pearson correlation coefficients are $\sim 0.98$ and $\sim 0.96$, respectively. Additionally, $\log \left(T_{\perp}\right)$ and $\log \left(T_{\|}\right)$are well-correlated (Pearson correlation coefficient $\sim 0.88$ ) in the slow solar wind. In the fast solar wind, the correlation coefficients are smaller than the corresponding coefficients in the slow solar wind. The largest correlation is still observed between $\log \left(T_{\perp}\right)$ and $\log (T)$, which gives a Pearson correlation coefficient of $\sim 0.94$. The coefficients between the other tensor elements are significantly smaller $(<0.64)$. We note that the number of analyzed data points in the fast solar wind $\left(V>500 \mathrm{kms}^{-1}\right)$ is significantly smaller than the number of analyzed data points in the slow solar wind $\left(V<500 \mathrm{kms}^{-1}\right.$; see the speed histogram in Figure 2). According to this result, we infer that the proton temperature measured along a single direction could 
be used successfully in the studies of some of the plasma thermodynamic properties. Such an application will be more accurate in the slow solar wind.
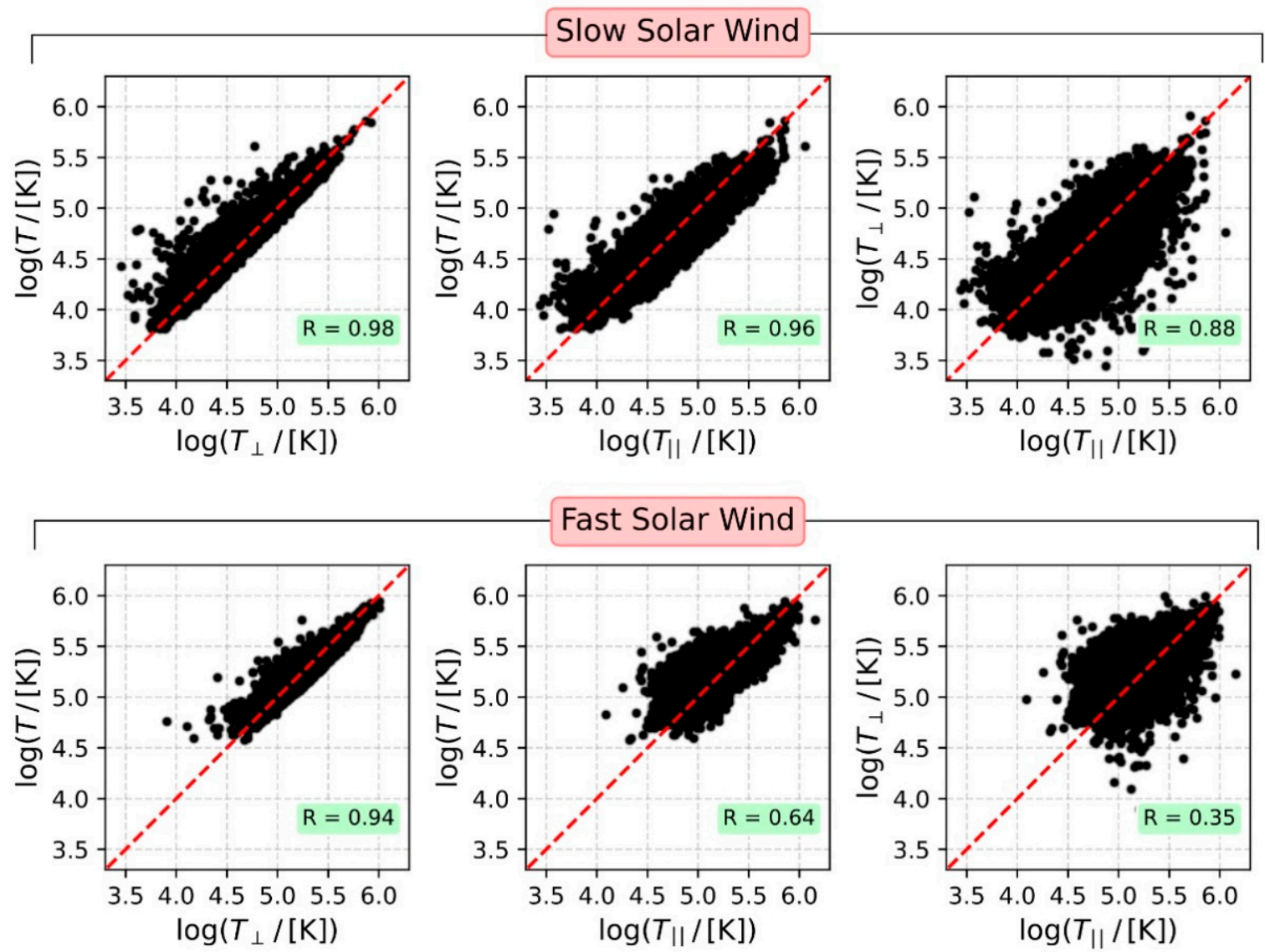

Figure 3. (Left) $\log (T)$ as a function of $\log \left(T_{\perp}\right)$; (middle) $\log (T)$ as a function of $\log \left(T_{\|}\right)$; and (right) $\log \left(T_{\perp}\right)$ as a function of $\log \left(T_{\|}\right)$for (top panels) slow solar wind with $V<500 \mathrm{kms}^{-1}$ and (bottom panels) for fast solar wind with $V>500 \mathrm{kms}^{-1}$. In each panel, we show the corresponding Pearson correlation coefficients between the plotted parameters, and the red dashed line shows the identity function.

We also investigate the long-term relationship between plasma density and temperature. In the left panel of Figure 4, we show the $2 \mathrm{D}$ histogram of $\log (n)$ and $\log (T)$. The middle panel of Figure 4 shows the $2 \mathrm{D}$ histogram of $\log (n)$ and $\log \left(T_{\perp}\right)$, while the right panel shows the $2 \mathrm{D}$ histogram of $\log (n)$ and $\log \left(T_{\|}\right)$. We normalize each column of the histograms to the maximum value within the column. Besides some obvious differences, the three histograms have a similar general structure. The large-scale fluctuations of $\log (n)$ and the logarithm of the temperature tensor elements are anti-correlated. Specifically, the general trend of the large-scale variations follow the grey dashed lines with a slope of -2 . However, we observe short-scale structures with positive slopes. Those appear as smaller, bright regions in the $2 \mathrm{D}$ histograms and may be relevant with the polytropic relationship that governs the plasma density and temperature fluctuations within plasma streamlines. We also observe that the histogram of $\log (n)$ and $\log (T)$ has many more similarities with the histogram of $\log (n)$ and $\log \left(T_{\perp}\right)$ than the histogram of $\log (n)$ and $\log \left(T_{\|}\right)$. 

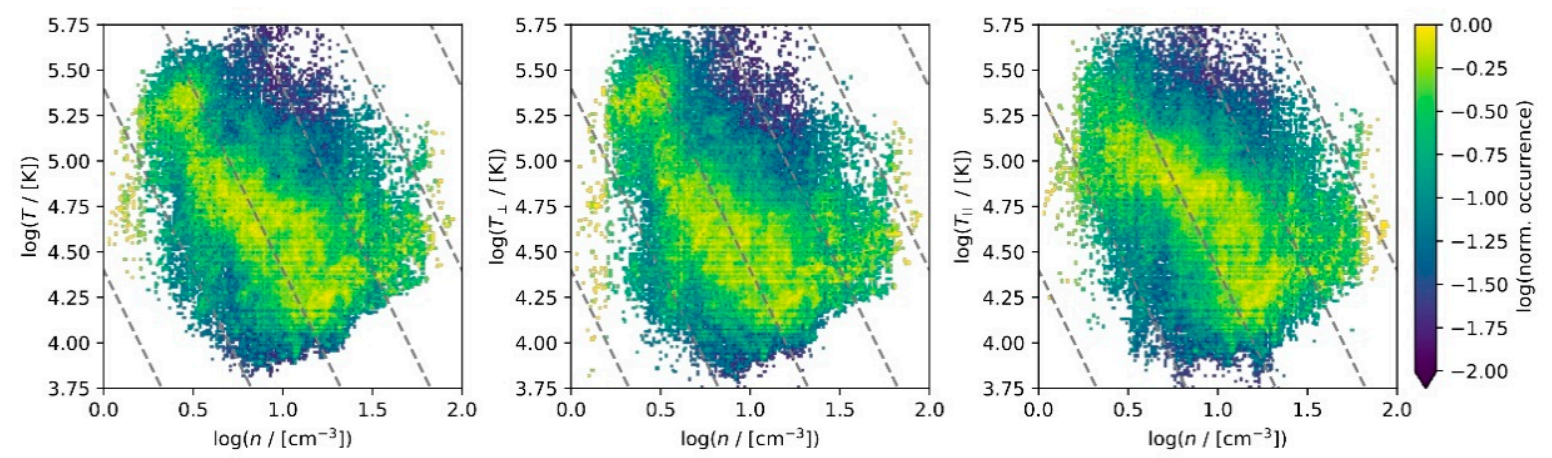

Figure 4. Two-dimensional histograms of (left) $\log (T)$ and $\log (n)$, (middle) $\log \left(T_{\perp}\right)$ and $\log (n)$, and (right) $\log \left(T_{\|}\right)$and $\log (n)$. The grey dashed lines show linear models with slopes -2 .

Following a similar technique as in [19-22,28,29], we determine $\gamma$ by fitting the polytropic model to $\ln (T)$ vs. $\ln (n)$ observations within short-scale subintervals with small $(<0.1)$ relative deviation of the Bernoulli integral. We further determine $\gamma_{\perp}$ and $\gamma_{\|}$from the slope of a linear fitting to $\ln \left(T_{\perp}\right)$ vs. $\ln (n)$ and $\ln \left(T_{\|}\right)$vs. $\ln (n)$, respectively. The left panel of Figure 5 shows the histograms of $\gamma, \gamma_{\perp}$ and $\gamma_{\|}$as derived from the 1995 observations. The right panel shows the histograms of the differences $\gamma_{\perp}-\gamma$ and $\gamma_{\|}-\gamma$. Table 1 shows the mean and standard deviation values for each histogram. Our results suggest that the linear regression between either $\log \left(T_{\perp}\right)$ and $\log (n)$, or $\log \left(T_{\|}\right)$and $\log (n)$, in selected short intervals of solar wind protons at $\sim 1$ au, with a quasi-stable Bernoulli integral, can estimate the average actual value of $\gamma$. The derived $\gamma_{\perp}$ is a better estimation of $\gamma$ than $\gamma_{\|}$. In the next section, we discuss our results in detail.
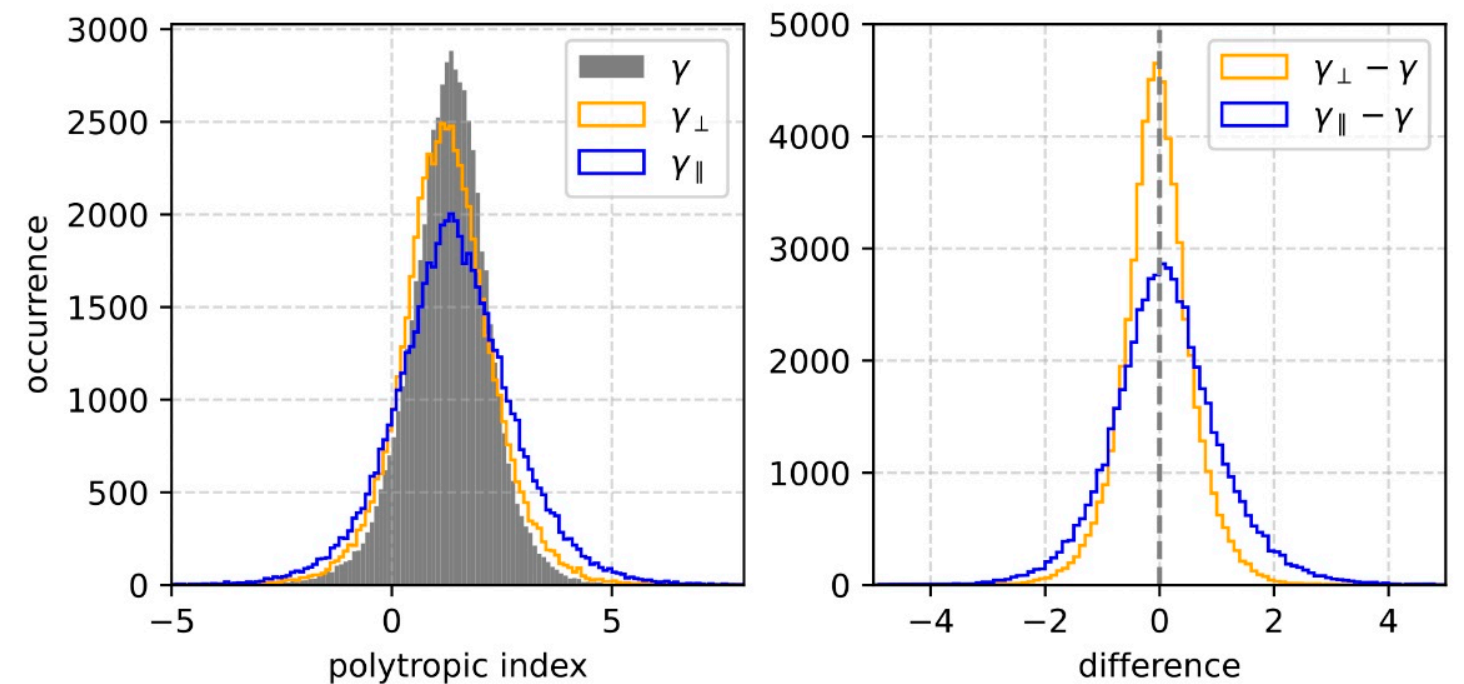

Figure 5. (Left) Histograms of (grey) the polytropic index $\gamma$, (orange) the index $\gamma_{\perp}$ as calculated by a linear regression to $\log \left(T_{\perp}\right)$ and $\log (n)$, and (blue) the index $\gamma_{\|}$, which is calculated by the linear regression to $\log \left(T_{\|}\right)$and $\log (n)$. (Right) Histograms of the differences (orange) $\gamma_{\perp}-\gamma$ and (blue) $\gamma_{\|}-\gamma$.

Table 1. Mean values and standard deviations of the histograms in Figure 5.

\begin{tabular}{cccccccccc}
\hline $\bar{\gamma}$ & $\sigma_{\bar{\gamma}}$ & $\bar{\gamma}_{\perp}$ & $\sigma_{\bar{\gamma}, \perp}$ & $\bar{\gamma}_{\|}$ & $\sigma_{\bar{\gamma}, \|}$ & $\overline{\gamma_{\perp}-\gamma}$ & $\sigma_{\overline{\left(\gamma_{\perp}-\gamma\right)}} \overline{\gamma_{\|}-\gamma}$ & $\sigma_{\overline{\left(\gamma_{\|}-\gamma\right)}}$ \\
\hline 1.30 & 0.93 & 1.26 & 1.08 & 1.41 & 1.39 & -0.04 & 0.62 & 0.11 & 0.97 \\
\hline
\end{tabular}




\section{Discussion}

We present analytical expressions of the relationships between $\log (T), \log \left(T_{\perp}\right)$ and $\log \left(T_{\|}\right)$. According to the analytical expressions, these relationships are linear for constant temperature anisotropy. Any anisotropy variations will affect the ordinate of the linear model. The ordinate of the linear model $\log (T)$ vs. $\log \left(T_{\perp}\right)$ is:

$$
A=\log \left(\frac{1+2 \alpha}{3 \alpha}\right)
$$

and we estimate its fluctuations, $\delta A$, due to the anisotropy fluctuations $\delta \alpha$, by using the error propagation formula:

$$
\delta_{A}=\left|\frac{\vartheta A}{\vartheta \alpha}\right| \delta_{\alpha}=\frac{\delta_{\alpha}}{\alpha(2 \alpha+1) \ln (10)}
$$

Similarly, the ordinate of the linear model $\log (T)$ vs. $\log \left(T_{\|}\right)$is:

$$
B=\log \left(\frac{1+2 \alpha}{3}\right)
$$

with fluctuations:

$$
\delta_{B}=\frac{2 \delta_{\alpha}}{(2 \alpha+1) \ln (10)} .
$$

Finally, the ordinate of the model $\log \left(T_{\perp}\right)$ vs. $\log \left(T_{\|}\right)$is:

$$
\Gamma=\log (\alpha),
$$

with fluctuations:

$$
\delta_{\Gamma}=\frac{\delta_{\alpha}}{\alpha \ln (10)} .
$$

The proton plasma parameters we analyze here have a typical temperature anisotropy $\alpha \sim 0.8$ (see Figure 2). For this anisotropy value, and according to Equations (10), (12) and (14), we find that $\delta_{A}<\delta_{B}<\delta_{\Gamma}$. This explains why the Pearson correlation coefficient is largest for $\log (T)$ vs. $\log \left(T_{\perp}\right)$ and smallest for $\log \left(T_{\perp}\right)$ vs. $\log \left(T_{\|}\right)$within the same plasma population we examine.

In Section 1, we show that the linear regression to $\log \left(T_{\perp}\right)$ vs. $\log (n), \operatorname{or} \log \left(T_{\|}\right)$vs. $\log (n)$ data can derive the plasma polytropic index only if the temperature anisotropy is constant within the analyzed streamlines. Notably, [30] show that there is a specific relationship between the polytropic index and the temperature anisotropy. Here, we refer to general variations in $a$ that could be statistical variations or measurement errors. If these fluctuations are significant within a streamline, the ordinate in the relationships $\log \left(T_{\perp}\right)$ vs. $\log (n)$, and $\log \left(T_{\|}\right)$vs. $\log (n)$ exhibits significant fluctuations as well, and the relationships are not linear. According to Equations (7) and (8), and the propagation of errors, we find that the ordinate fluctuations in $\log \left(T_{\perp}\right)$ vs. $\log (n)$ are $\delta_{A}$, exactly as given in Equation (10). The ordinate fluctuations in $\log \left(T_{\|}\right)$vs. $\log (n)$ are $\delta_{B}$, given by Equation (12). Therefore, we expect that the linear fitting to $\log \left(T_{\perp}\right)$ vs. $\log (n)$ derives the polytropic index more accurately than the linear fitting to $\log \left(T_{\|}\right)$vs. $\log (n)$. The 2D histograms of the plasma temperature and density in Figure 4 verify this expectation, showing that the relationship between $\log \left(T_{\perp}\right)$ and $\log (n)$ has many more similarities to the relationship between $\log (T)$ and $\log (n)$ than the relationship between $\log \left(T_{\|}\right)$and $\log (n)$. This is not surprising, considering also the definition of $T$ in Equation (1) and that the typical anisotropy value is 0.8 , meaning that $T_{\perp} \sim 0.8 T_{\|}$. 
All three histograms in Figure 4 show evidence that the large-scale variations of the plasma density and temperature follow a linear model $\log (T) \propto-2 \log (n)$. Newbury et al. [31] discuss the specific relationship which is apparent in the ambient solar wind and reflects the differences in the solar wind origins, and that it is not necessarily relevant to the solar wind expansion. On the other hand, the short-scale fluctuations of the scalar, perpendicular, and parallel temperature that we observe in the 2D histograms are positively correlated with the plasma density fluctuations. This positive correlation is possibly due to the polytropic behavior of the plasma protons within plasma streamlines.

We derive the polytropic index $\gamma$ of the protons by analyzing short subintervals $(<15 \mathrm{~min})$ in 1995. Our analysis derives an average value $\bar{\gamma} \sim 1.3$ and a standard deviation $\sigma_{\bar{\gamma}} \sim 0.93$. The analysis of [22] derives an average $\gamma \sim 1.45$ for the 1995 observations. The difference is possibly due to the different filters applied to the selected data and the duration of the analyzed subintervals. Finally, we derive the polytropic index by fitting a linear model to the fluctuations of $T_{\perp}$ and $T_{\|}$. The average polytropic index values we find with these regressions are $\bar{\gamma}_{\perp} \sim 1.26$ and $\bar{\gamma}_{\|} \sim 1.41$, with standard deviations $\sigma_{\bar{\gamma}, \perp} \sim 1.08$ and $\sigma_{\bar{\gamma}, \|} \sim 1.39$, respectively. Therefore, according to our statistical study, we derive similar (within $~ 0.1$ ) average polytropic index values regardless of the temperature tensor element we use in our linear regression analysis. We infer that the anisotropic heating within the analyzed streamlines is not significant enough to prevent accurately calculating the average polytropic index of the solar wind plasma protons. We also find that $\sigma_{\bar{\gamma}}<\sigma_{\bar{\gamma}, \perp}<\sigma_{\bar{\gamma}, \perp}$, which is in agreement with the expected deviations from the linear model that we discuss above. We acknowledge that this result is based on the specific streamline selection criteria. For instance, if we analyze longer subintervals, we will accumulate larger anisotropy fluctuations within the analyzed data. That will reduce the accuracy of the polytropic indices that are calculated with either $T_{\perp}$ or $T_{\|}$instead of $T$.

Finally, we emphasize that our study focuses on solar wind protons at 1 au. It will be of a great interest to examine the same mechanism in solar wind electrons, or even in other solar wind ion species through the heliosphere. The recent ESA's Solar Orbiter mission is determined to provide high-quality measurements of electron and heavy ion 3D VDFs. These measurements will be ideal to investigate the temperature anisotropy and the polytropic behavior of solar wind species. As has been recently shown by [22,32,33], studies of the polytropic behavior in space and astrophysical plasmas leads to important knowledge about the velocity distribution functions of the plasma particles and the potential energy acting on them.

\section{Conclusions}

In summary, we find that:

- The long-term fluctuations of the proton temperature tensor elements are very well correlated in the slow solar wind at $\sim 1 \mathrm{au}$;

- In fast solar wind protons, the scalar temperature is very well-correlated only with the perpendicular temperature;

- Fittings to the fluctuations of proton density and any of the proton temperature tensor elements within short intervals ( $<15 \mathrm{~min}$ ) derive, on average, very similar polytropic indices;

- The perpendicular temperature derives the polytropic index more accurately than the parallel temperature does, which is expected considering the definition of the scalar temperature and the typical value of solar wind temperature anisotropy.

Author Contributions: Conceptualization, G.N.; methodology, G.N.; software, G.N.; validation, G.N. and G.L.; formal analysis, G.N.; investigation, G.N. and G.L.; resources, M.I.D.; data curation, G.N.; writing—original draft preparation, G.N.; writing—review and editing, G.N., G.L. and M.I.D.; visualization, G.N.; supervision, G.L. and M.I.D.; project administration, M.I.D.; funding acquisition, M.I.D. All authors have read and agreed to the published version of the manuscript.

Funding: This research was funded by NASA, Grant 80NSSC21K0112. 
Institutional Review Board Statement: Not applicable.

Informed Consent Statement: Not applicable.

Data Availability Statement: The dataset we use in this study is publicly available at https:// cdaweb.gsfc.nasa.gov / index.html (accessed on 19 May 2020).

Acknowledgments: G.N. thanks Daniel Verscharen and Robert T. Wicks for the helpful discussions.

Conflicts of Interest: The authors declare no conflict of interest.

\section{References}

1. Gary, S.P. Theory of Space Plasma Microinstabilities; Cambridge University Press: Cambridge, UK, 1993.

2. Marsch, E. Kinetic Physics of the Solar Wind. Living Rev. Sol. Phys. 2006, 3, 1. [CrossRef]

3. Verscharen, D.; Klein, K.G.; Maruca, B.A. The multi-scale nature of the solar wind. Living Rev. Sol. Phys. 2019, 16, 5. [PubMed]

4. Olsen, E.L.; Leer, E. A study of solar wind acceleration based on gyrotropic transport equations. J. Geophys. Res. 1999, 104, 9963-9973. [CrossRef]

5. Nicolaou, G.; Wicks, R.T.; Livadiotis, G.; Verscharen, D.; Owen, C.J.; Kataria, D.O. Determining the bulk parameters of plasma electrons from pitch-angle distribution measurements. Entropy 2020, 22, 103. [CrossRef] [PubMed]

6. Liu, Y.; Richardson, J.D.; Belcher, J.W.; Kasper, J.C. Temperature Anisotropy in a Shocked Plasma: Mirror-Mode instabilities in the Heliosheath. Astrophys. J. 2007, 659, L65. [CrossRef]

7. Bader, A.; Stenberg Wieser, G.; André, M.; Wieser, M.; Futaana, Y.; Persson, M.; Nilsson, H.; Zhang, T.L. Proton Temperature Anisotropies in the Plasma Environment of Venus. J. Geophys. Res. 2019, 124, 3312-3330. [CrossRef]

8. Hietala, H.; Drake, J.F.; Phan, T.D.; Eastwood, J.P.; McFadden, J.P. Ion temperature anisotropy across a magnetotail reconnection jet. J. Geophys. Res. 2015, 42, 7239-7247. [CrossRef]

9. Case, A.W.; Kasper, J.C.; Stevens, M.L.; Korreck, K.E.; Paulson, K.; Daigneau, P.; Caldwell, D.; Freeman, M.; Henry, T.; Klingensmith, B.; et al. The Solar Probe Cup on the Parker Solar Probe. Astrophys. J. 2020, 246, 43. [CrossRef]

10. Parker, E.N. Interplanetary Dynamical Processes; Wiley-Interscience: Hoboken, NJ, USA, 1963.

11. Chandrasekhar, S. An Introduction to the Study of Stellar Structure; Dover Publications: New York, NY, USA, 1967.

12. Bavassano, B.; Bruno, R.; Rosenbauer, H. Compressive fluctuations in the solar wind and their polytropic index. Annal. Geophys. 1996, 14, 510-517. [CrossRef]

13. Kartalev, M.; Dryer, M.; Grigorov, K.; Stoimenova, E. Solar wind polytropic index estimates based on single spacecraft plasma and interplanetary magnetic field measurements. J. Geophys. Res. 2006, 111, A10107. [CrossRef]

14. Arridge, C.S.; McAndrews, H.J.; Jackman, C.M.; Forsyth, C.; Walsh, A.P.; Sittler, E.C.; Gilbert, L.K.; Lewis, G.R.; Russell, C.T.; Coates, A.J.; et al. Plasma electrons in Saturn's magnetotail: Structure, distribution and energisation. Planet. Space Sci. 2009, 57, 2032-2047. [CrossRef]

15. Dialynas, K.; Roussos, E.; Regoli, L.; Paranicas, C.P.; Krimigis, S.M.; Kane, M.; Mitchell, D.G.; Hamilton, D.C.; Krupp, N.; Carbary, J.F. Energetic ion moments and polytropic index in Saturn's magnetosphere using Cassini/MIMI measurements: A simple model based on k-distribution functions. J. Geophys. Res. 2018, 123, 8066-8086. [CrossRef]

16. Nicolaou, G.; McComas, D.J.; Bagenal, F.; Elliott, H.A. Properties of plasma ions in the distant Jovian magnetosheath using Solar Wind Around Pluto data on New Horizons. J. Geophys. Res. 2014, 119, 3463-3479. [CrossRef]

17. Nicolaou, G.; McComas, D.J.; Bagenal, F.; Elliott, H.A.; Ebert, R.W. Jupiter's deep magnetotail boundary layer. Planet. Space Sci. 2015, 111, 116-125. [CrossRef]

18. Totten, T.L.; Freeman, J.W.; Arya, S. An empirical determination of the polytropic index for the free-streaming solar wind using Helios 1 data. J. Geophys. Res. 1995, 100, 13-17. [CrossRef]

19. Nicolaou, G.; Livadiotis, G.; Wicks, R.T.; Verscharen, D.; Maruca, B.A. Polytropic Behavior of Solar Wind Protons Observed by Parker Solar Probe. Astrophys. J. 2020, 901, 26. [CrossRef]

20. Nicolaou, G.; Livadiotis, G.; Moussas, X.D. Long-Term Variability of the Polytropic Index of Solar Wind Protons at 1 AU. Sol. Phys. 2014, 289, 1371-1378. [CrossRef]

21. Livadiotis, G. Long-term independence of solar wind polytropic index on plasma flow speed. Entropy 2018, 20, 799. [CrossRef]

22. Nicolaou, G.; Livadiotis, G. Long-term Correlations of Polytropic Indices with Kappa Distributions in Solar Wind Plasma near 1 au. Astrophys. J. 2019, 884, 52. [CrossRef]

23. Elliott, H.A.; McComas, D.J.; Zirnstein, E.J.; Randol, B.M.; Delamere, P.A.; Livadiotis, G.; Bagenal, F.; Barnes, N.P.; Stern, A.S.; Young, L.A.; et al. Slowing of the Solar Wind in the Outer Heliosphere. Astrophys. J. 2019, 885, 156. [CrossRef]

24. Livadiotis, G.; McComas, D.J.; Schwadron, N.A.; Funsten, H.O.; Fuselier, S.A. Pressure of the proton plasma in the inner heliosheath. Astrophys. J. 2013, 762, 134. [CrossRef]

25. Livadiotis, G. Radial Profile of the Polytropic Index of Solar Wind Plasma in the Heliosphere. Res. Notes AAS 2021, 5, 4. [CrossRef]

26. Lepping, R.P.; Acũna, M.H.; Burlaga, L.F.; Farrel, W.M.; Slavin, J.A.; Schatten, K.H.; Mariani, F.; Ness, N.F.; Neubauer, F.M.; Whang, Y.C.; et al. The WIND magnetic field investigation. Space Sci. Rev. 1995, 71, 207-229. [CrossRef]

27. Kasper, J. Solar Wind Plasma: Kinetic Properties and Micro-Instabilities. Ph.D. Thesis, Massachusetts Institute of Technology, Cambridge, MA, USA, 2003. 
28. Nicolaou, G.; Livadiotis, G.; Wicks, R.T. On the Calculation of the Effective Polytropic Index in Space Plasmas. Entropy 2019, 21, 997. [CrossRef]

29. Livadiotis, G.; Desai, M.I. Plasma-field coupling at small length scales in solar wind near 1 au. Astrophys. J. 2016, 829, 88. [CrossRef]

30. Livadiotis, G.; Nicolaou, G. Relationship between polytropic index and temperature anisotropy in space plasmas. Astrophys. J. 2021, 909, 127. [CrossRef]

31. Newbury, J.A.; Russel, C.T.; Lindsay, G.M. Solar wind polytropic index in the vicinity of stream interactions. Geophys. Res. Lett. 1997, 24, 1431-1434. [CrossRef]

32. Livadiotis, G. On the Origin of Polytropic Behavior in Space and Astrophysical Plasmas. Astrophys. J. 2019, 874, 10. [CrossRef]

33. Livadiotis, G. Using Kappa Distributions to Identify the Potential Energy. J. Geophys. Res. 2018, 123, 1050-1060. [CrossRef] 